\title{
openheart Gender differences in the decrease of in-hospital mortality in patients with acute myocardial infarction during the last 20 years in Switzerland
}

\author{
Dragana Radovanovic, ${ }^{1}$ Burkhardt Seifert, ${ }^{2}$ Marco Roffi, ${ }^{3}$ Philip Urban, ${ }^{4}$ \\ Hans Rickli, ${ }^{5}$ Giovanni Pedrazzini, ${ }^{6}$ Paul Erne ${ }^{1,7}$
}

To cite: Radovanovic D, Seifert B, Roffi M, et al. Gender differences in the decrease of in-hospital mortality in patients with acute myocardial infarction during the last 20 years in Switzerland. Open Heart 2017;4:e000689. doi:10.1136/ openhrt-2017-000689

Received 20 July 2017 Revised 23 August 2017 Accepted 4 October 2017

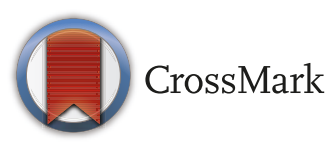

${ }^{1}$ AMIS Plus Data Center, Epidemiology, Biostatistics and Prevention Institute, University of Zurich, Zurich, Switzerland ${ }^{2}$ Department of Biostatistics, Epidemiology, Biostatistics and Prevention Institute, University of Zurich, Zurich, Switzerland ${ }^{3}$ Division of Cardiology, University Hospital Geneva, Geneva, Switzerland

${ }^{4}$ Cardiology Department, La Tour Hospital, Geneva, Switzerland

${ }^{5}$ Department of Cardiology, Kantonsspital St. Gallen, St. Gallen, Switzerland

${ }^{6}$ Department of Cardiology, Cardiocentro Ticino, Lugano, Switzerland

${ }^{7}$ Department of Biomedicine, University of Basel, Basel, Switzerland

Correspondence to Dr Dragana Radovanovic; dragana.radovanovic@uzh.ch

\section{ABSTRACT}

Objective To assess temporal trends of in-hospital mortality in patients with acute myocardial infarction (AMI) enrolled in the Swiss nationwide registry (AMIS Plus) over the last 20 years with regard to gender, age and inhospital treatment.

Methods All patients with AMl from 1997 to 2016 were stratified according to ST-segment elevation myocardial infarction (STEMI) or non-STEMI (NSTEMI), and gender using logistic regression analyses.

Results Among 51725 patients, 30398 (59\%) had STEMI and 21327 (41\%) had NSTEMl; 73\% were men (63.9 \pm 12.8 years) and $27 \%$ were women $(71.7 \pm 12.5$ years). Over 20 years, crude in-hospital STEMI mortality decreased from $9.8 \%$ to $5.5 \%$ in men and from $18.3 \%$ to $6.9 \%$ in women. In patients with NSTEMI, it decreased from $7.1 \%$ to $2.1 \%$ in men and from $11.0 \%$ to $3.6 \%$ in women. After adjustment for age, mortality decreased per additional admission year by $3 \%$ in men with STEMI (OR $0.97,95 \% \mathrm{Cl} 0.96$ to $0.98, \mathrm{P}<0.001$ ), by $5 \%$ in women with STEMI (OR $0.95,95 \% \mathrm{Cl} 0.93$ to $0.96, \mathrm{P}<0.001$ ), by $6 \%$ in men with NSTEMI (OR $0.94,95 \% \mathrm{Cl} 0.93$ to 0.96 , $\mathrm{P}<0.001)$ and by $5 \%$ in women with NSTEMI (OR 0.95, $95 \% \mathrm{Cl} 0.93$ to $0.97, \mathrm{P}<0.001$ ). In patients $<60$ years, a decrease in mortality was seen in women with STEMI (OR $0.94,95 \% \mathrm{Cl} 0.90$ to $0.99, \mathrm{P}=0.025$ ) and NSTEMI (OR $0.87,95 \% \mathrm{Cl} 0.80$ to $0.94, \mathrm{P}<0.001$ ) but not in men with STEMI (OR 1.01, 95\% Cl 0.98 to $1.04, \mathrm{P}=0.46$ ) and NSTEM (OR $0.98,95 \% \mathrm{Cl} 0.94$ to $1.03, \mathrm{P}=0.41)$. The mortality decrease in patients with AMI was closely associated with the increase in reperfusion therapy.

Conclusion From 1997 to 2016, in-hospital mortality of patients with AMI in Switzerland has halved and was more pronounced in women, particularly in the age category $<60$ years.

Trial registration number NCT01305785; Results.

\section{INTRODUCTION}

Cardiovascular disease continues to be the leading cause of death in the Western world in both women and men. Administrative data showed that mortality of patients with acute myocardial infarction (AMI) considerably decreased worldwide between the 1970s and the beginning of the new millennium. ${ }^{1-7}$

\section{KEY QUESTIONS}

What is already known about this subject? Women with acute myocardial infarction (AMI) have higher in-hospital mortality. However, an overal decrease in mortality has been reported in studies using administrative databases.

What does this study add?

These contemporary clinical data reveal that the gender gap in AMI mortality has been reduced over the past two decades, particularly in young women. AMI therapy has undergone substantial changes. The mortality decrease could in large be explained by the increase in reperfusion therapy.

How might this impact on clinical practice? These results are important for cardiovascular epidemiology and cardiologists. The trends in treatment and in-hospital mortality of patients with AMl in Switzerland are positive. Trends should be closely monitored in general to enable a timely reaction if needed, particularly for certain patient subgroups.

The lower risk of AMI in women cannot be explained by differences in established coronary heart disease risk factors. ${ }^{8}$ However, important differences in baseline characteristics between male and female patients with AMI have been published in our previous studies. $^{9-11}$ Further reports showed that younger women with acute coronary syndrome (ACS) had worse outcomes than men of the same age. ${ }^{72-15}$

Although women with AMI have a higher mortality than men, a prominent decline has occurred since 2000 , which could be the result of the application of evidence-based therapies and education to improve awareness of heart disease in women. ${ }^{16}$ Most of the published data only cover the time period up until 2008, and knowledge on mortality trends in hospital mortality of patients with AMI in Switzerland is sparse, revealing the 
need for a study that includes contemporary clinical data with a long-lasting observational period.

Therefore, the aims of this study were to assess gender differences in in-hospital mortality of patients with ST-segment elevation myocardial infarction (STEMI) and non-STEMI (NSTEMI) during the last 20 years in Switzerland and to evaluate these differences in patients below 60 years of age.

\section{METHODS}

The AMIS Plus registry is an ongoing nationwide prospective cohort of patients admitted with ACS to hospitals in Switzerland, founded by the Swiss Societies of Cardiology, Internal Medicine and Intensive Care Medicine in 1997. Details of the registry have been described elsewhere. ${ }^{17-20}$ Since 1997, 83 hospitals ranging from community institutions to large tertiary facilities temporarily or continuously provide, on a voluntary basis, blinded data for each patient by the treating physician or a trained study nurse through standardised internet-based or paperbased questionnaires. All data are checked for completeness, plausibility and consistency by the AMIS Plus Data Center (Epidemiology, Biostatistics and Prevention Institute, University of Zurich, Switzerland). The data centre queries treating physicians or study nurses if data are incomplete, implausible or inconsistent. Since 2010, external monitoring is regularly performed in randomly selected hospitals using randomly selected cases.

\section{Patient selection}

The present study included all patients with a definitive diagnosis of AMI defined according to the universal definitions of myocardial infarction (MI) by characteristic symptoms and/or ECG changes and/or cardiac marker elevation (either creatine kinase MB fraction at least two times the upper limit of normal or troponin I or $\mathrm{T}$ above individual hospital cut-off levels for MI). ${ }^{21}$ Patients included in this analysis were categorised as having STEMI or NSTEMI based on the initial ECG findings. Classification of STEMI included evidence of AMI as described above and ST segment elevation and/or new left bundle branch block (LBBB) on the initial ECG. NSTEMI included patients with ischaemic symptoms, ST segment depression or T-wave abnormalities in the absence of ST elevation on the initial ECG. All patients with a definitive diagnosis of AMI were included regardless of age, delay, inpatient onset or therapy received, and only patients with unstable angina were excluded.

Reperfusion therapy included thrombolysis or percutaneous coronary intervention (PCI). Immediate drug therapy was defined if administrated within 24 hours after presentation and was prescribed according to current practice and guideline recommendations at the time of the index presentations. The choice of intervention and medication was at the discretion of the treating physicians and hence reflects common practice in Switzerland.

\section{Statistical analyses}

The results are presented as percentages for categorical variables and were analysed using the Pearson $\chi^{2}$ test or Fisher's exact test as appropriate. Continuous normally distributed variables are expressed as means $\pm 1 \mathrm{SD}$ and were compared using the Student's two-tailed unpaired t-test. Continuous non-normally distributed variables are expressed as medians and IQRs and were analysed using the Mann-Whitney U test.

Univariable and multivariable binary logistic regressions were used to analyse trends of mortality over admission years. The explanatory variables were gender, age and therapies. Admission year was included as a covariate. As the effect of age on mortality was non-linear, a quadratic dependence of age was used in multivariable regression. Model fit was assessed using the Hosmer-Lemeshow test. Adjusted ORs are reported with 95\% CIs. The use of therapies changed non-linearly over time. Hence, gender differences in therapies were stratified by year of admission and analysed using the Mantel-Haenszel test. Interactions were assessed using the Breslow-Day test. A $P$ value of less than 0.01 was considered significant. IBM SPSS Statistics software (V.23, IBM Corp. Armonk, NY, USA) was used for statistical analyses.

\section{RESULTS}

From January 1997 to December 2016, 51725 patients with AMI from 83 Swiss hospitals were enrolled in the AMIS Plus registry and included in this study. Among them, 30398 (59\%) had STEMI and 21327 (41\%) had NSTEMI; $73 \%$ were men (mean age $63.9 \pm 12.8$ years) and $27 \%$ were women $(71.7 \pm 12.5$ years $)$.

The probability of in-hospital mortality using logistic regression in patients with AMI aged 66 years according to admission year and gender is depicted in figure 1A,B.

\section{ST-segment elevation myocardial infarction}

Of the patients with STEMI, 26\% were women aged $71.3 \pm 12.7$ years compared with men aged $62.8 \pm 12.8$ years $(\mathrm{P}<0.001)$. Over the study period, crude in-hospital mortality decreased from $9.8 \%$ to $5.5 \%$ in men and from $18.3 \%$ to $6.9 \%$ in women (both $\mathrm{P}<0.001$ ) (figure 2 ). The quadratic model of logistic regression for mortality of patients with STEMI demonstrated a significance for non-linearity of age of $\mathrm{P}<0.001$. After adjustment for gender and age, early mortality reduction was $4 \%$ per additional admission year for all patients with STEMI (OR $0.96,95 \%$ CI 0.95 to $0.97, \mathrm{P}<0.001$ ). There was a weak interaction between admission year and gender $(\mathrm{P}=0.019)$. Age-adjusted mortality decreased in men with STEMI at a rate of $3 \%$ (OR $0.97,95 \%$ CI 0.96 to 0.98 , $\mathrm{P}<0.001)$ and in STEMI women at a rate of $5 \%$ per additional admission year (OR $0.95,95 \%$ CI 0.93 to 0.96 , $\mathrm{P}<0.001)$.

\section{Non-ST-segment elevation myocardial infarction}

Among patients with NSTEMI, 27\% were women and were older than men $(72.2 \pm 12.2$ years vs $65.6 \pm 12.6$ 

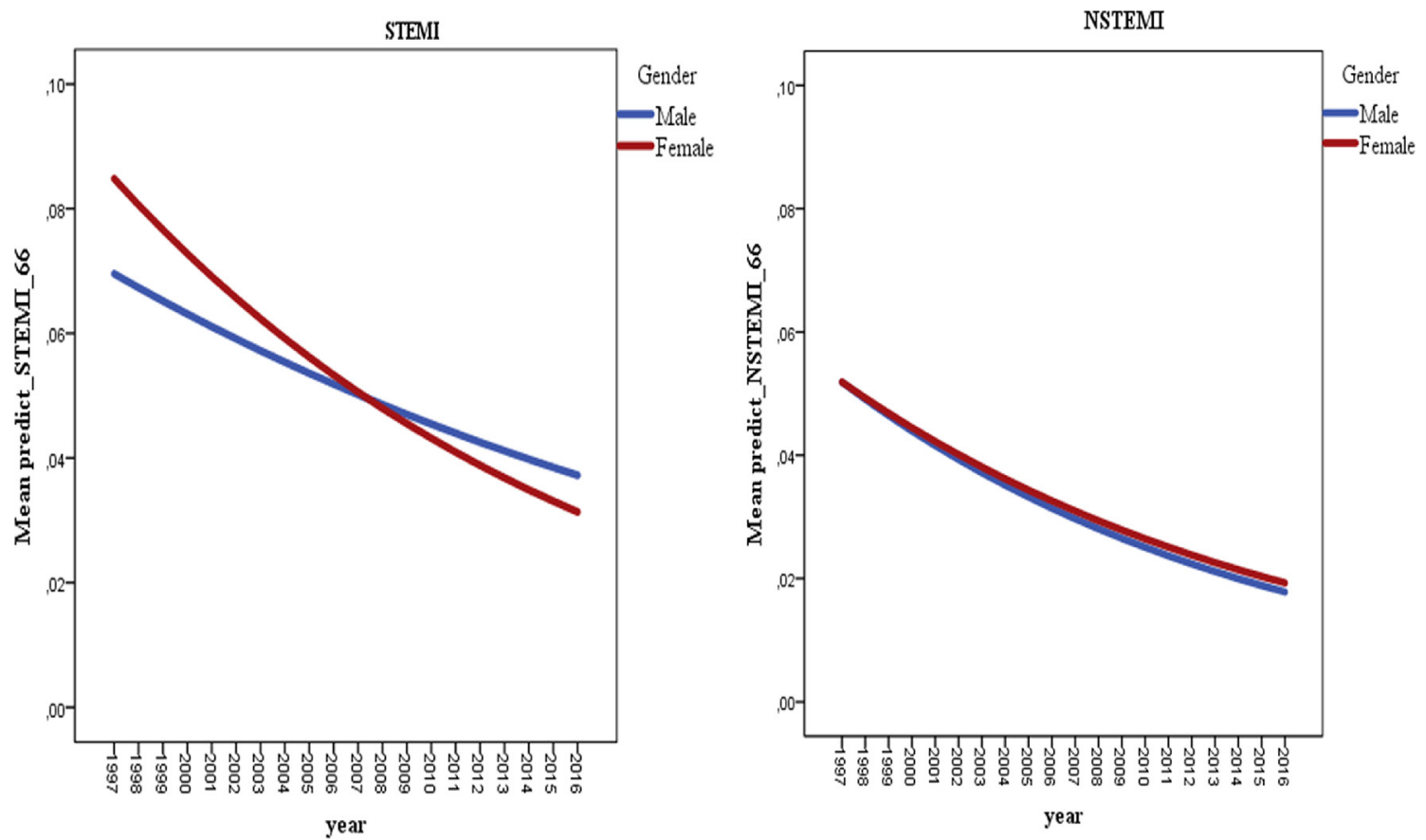

Figure 1 The predicted probability of in-hospital mortality in patients with AMI aged 66 years according to admission year and gender. STEMI, ST-segment elevation myocardial infarction; NSTEMI, non-ST-segment elevation myocardial infarction.

years, $\mathrm{P}<0.001)$. From 1997 to 2016, crude in-hospital mortality decreased in men from $7.1 \%$ to $2.1 \%(\mathrm{P}<0.001)$ and in women from $11.0 \%$ to $3.6 \%(\mathrm{P}<0.001)$ (figure 3$)$. The quadratic model of logistic regression for mortality of patients with NSTEMI also demonstrated a significance for non-linearity of age $(\mathrm{P}=0.001)$. After adjustment for gender and age, early mortality reduction was $6 \%$ for all patients with NSTEMI (OR 0.94, 95\% CI 0.93 to 0.96 , $\mathrm{P}<0.001)$ with no interaction between admission year and gender $(\mathrm{P}=0.75)$. The age-adjusted mortality decrease per additional admission year in patients with NSTEMI was even higher at $6 \%$ in men (OR 0.94, $95 \%$ CI 0.93 to $0.96, \mathrm{P}<0.001)$ and $5 \%$ in women (OR $0.95,95 \%$ CI 0.93 to $0.97, \mathrm{P}<0.001)$.

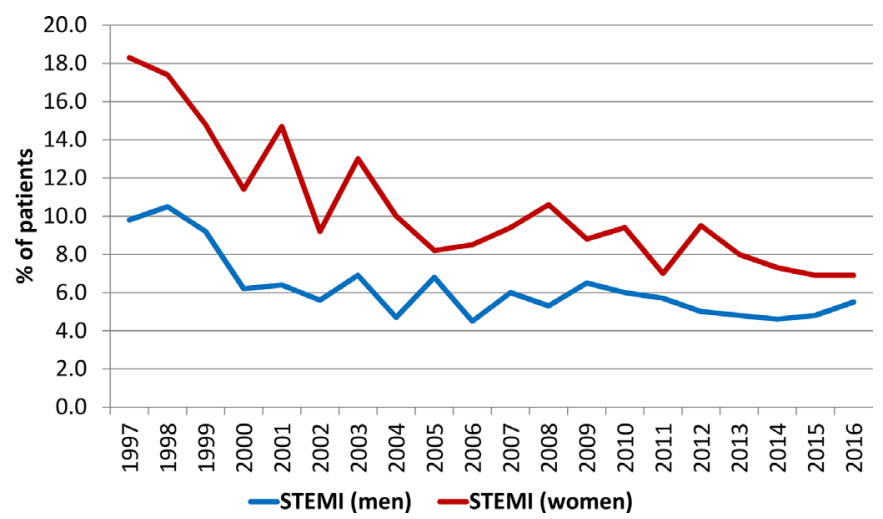

Figure 2 Crude in-hospital mortality of patients with STEMI according to admission year and gender from 1997 to 2016. STEMI, ST-segment elevation myocardial infarction.

\section{Reperfusion}

The use of reperfusion (thrombolysis or PCI) increased in male patients with STEMI from $60 \%$ to $93 \%(\mathrm{P}<0.001)$ and in female patients with STEMI from $45 \%$ to $90 \%$ $(\mathrm{P}<0.001)$. Use of thrombolysis in patients with STEMI continuously decreased from $47 \%$ in 1997 down to $0.2 \%$ in $2016(\mathrm{P}<0.001)$, whereby the use of PCI increased from $8.3 \%$ to $91.8 \%(\mathrm{P}<0.001)$. This increase between 1997 and 2016 was gender dependent (interaction between admission year and gender, $\mathrm{P}<0.001)$ : in men from $9.1 \%$ to $92.5 \%(\mathrm{P}<0.001)$ and in women from $6.0 \%$ to $89.5 \%$ $(\mathrm{P}<0.001)$.

The use of PCI increased in patients with NSTEMI from $3.8 \%$ to $75.8 \%(\mathrm{P}<0.001)$. This increase between 1997 and 2016 was not gender dependent (interaction between

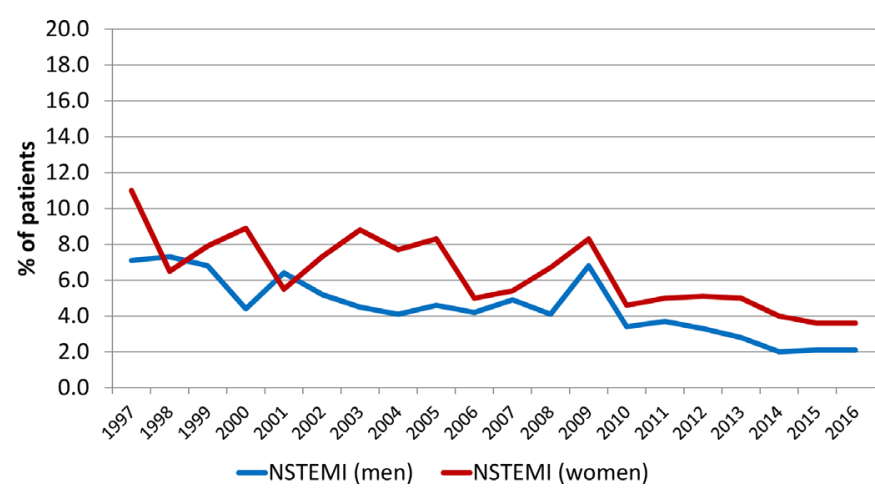

Figure 3 Crude in-hospital mortality of patients with NSTEMI according to admission year and gender from 1997 to 2016. NSTEMI, non-ST-segment elevation myocardial infarction. 
admission year and gender, $\mathrm{P}=0.42)$ : in men from $4.7 \%$ to $76.5 \%(\mathrm{P}<0.001)$ and in women from $1.4 \%$ to $74.5 \%$ $(\mathrm{P}<0.001)$.

The mortality decrease in patients with AMI could in large be explained by the increase in reperfusion therapy: after adjustment for reperfusion use, a reduction in mortality of $1 \%$ per year remained (OR $0.99,95 \%$ CI 0.98 to $1.00, \mathrm{P}=0.007)$, whereas the gender difference disappeared (OR $0.99,95 \%$ CI 0.90 to $1.09, \mathrm{P}=0.85$ ).

\section{Drug therapies}

Throughout the entire observational period, women with AMI (STEMI and NSTEMI) were overall less likely to receive guideline-recommended drugs than men with AMI. Among women, $94.0 \%$ received aspirin versus $95.6 \%$ of men (OR $0.72,95 \%$ CI 0.66 to $0.78, \mathrm{P}<0.001$ ) with no evidence of OR changes for gender over time (Breslow-Day $\mathrm{P}=0.80$ ). Overall P2Y12 inhibitors (clopidogrel, prasugrel or ticagrelor) were given to $63.8 \%$ of women compared with $72.3 \%$ of men (OR $0.61,95 \%$ CI 0.58 to $0.65, \mathrm{P}<0.001$ ) with significant $\mathrm{OR}$ changes during the observation period (Breslow-Day $\mathrm{P}=0.009$ ). Statins were given to $69.2 \%$ of women compared with $76.5 \%$ of men (OR $0.69,95 \%$ CI 0.66 to $0.73, \mathrm{P}<0.001$ ) with no OR changes over time (Breslow-Day $\mathrm{P}=0.24$ ). Beta blockers were given to $61.4 \%$ of female patients with AMI versus $64.6 \%$ of male patients (OR $0.86,95 \%$ CI 0.82 to $0.90, \mathrm{P}<0.001$ ) with OR changes over time (Breslow-Day $\mathrm{P}<0.001)$ and angiotensin-converting enzyme inhibitors/ angiotensin II receptor antagonists to $49.3 \%$ of women versus $50.8 \%$ of men (OR $0.95,95 \%$ CI 0.92 to 0.98 , $\mathrm{P}=0.021$ ) without significant $\mathrm{OR}$ changes during the observational years (Breslow-Day $\mathrm{P}=0.021$ ).

\section{Younger patients with AMI}

In patients aged below 60 years, a decrease in early mortality per additional admission year of STEMI was seen in women (OR $0.94,95 \%$ CI 0.90 to $0.99, \mathrm{P}=0.025)$ but not in men (OR 1.01, 95\% CI 0.98 to $1.04, \mathrm{P}=0.46$ ) (interaction between gender and admission year $\mathrm{P}=0.019$ ). Even greater differences were seen in patients with NSTEMI. Mortality per year dramatically decreased in NSTEMI women (OR $0.87,95 \%$ CI 0.80 to $0.94, \mathrm{P}<0.001)$ but was not significant in men (OR 0.98, 95\% CI 0.94 to 1.03, $\mathrm{P}=0.41$ ) (interaction between gender and admission year, $\mathrm{P}=0.006)$.

\section{DISCUSSION}

The results of this study show that over the last 20 years, in-hospital mortality of patients with AMI in Switzerland has halved and the gender gap reduced. Although in-hospital mortality was consistently higher in women, overall age-adjusted mortality has decreased more prominently in women compared with men. In this contemporary cohort of 51725 patients, a reduction in mortality was seen in patients with STEMI and NSTEMI of both genders. However, in patients aged below 60 years, a significant decrease in in-hospital mortality was observed in women but not in men.

The gender differences in the baseline characteristics of patients with AMI are well known and repeatedly described in the literature: women are older, have a higher burden of risk factors, have more comorbidities and are less likely to receive evidence-based therapies. ${ }^{1622-25}$ Previously published works of ours have also shown this. ${ }^{926}$

However, gender differences in short-term and longterm crude mortality of patients with AMI have been described in several studies with contradictory results. Studies mainly reported on gender differences in crude mortality, but the differences no longer existed if mortality was adjusted for age, risk factors or comorbidities. ${ }^{124} 2728$ Analyses of 78254 patients from 2001 to 2006 in the 'Get with the Guidelines' database showed no differences in in-hospital mortality of all patients with AMI, but it was higher in women with STEMI. ${ }^{25}$ Women with ACS had worse in-hospital outcomes than men, ${ }^{22}$ and age-adjusted in-hospital mortality was higher for women. ${ }^{29}$ Analysis of temporal trends in in-hospital mortality of 916380 patients with AMI from 1994 to 2006 in the National Registry of Myocardial Infarction showed that women experienced larger improvements than men. ${ }^{30}$ This is in line with our current results.

AMI therapy has undergone substantial changes over the 20-year period, and the use of reperfusion therapies has continually increased away from thrombolysis towards PCI, but there was a significant underuse in women. ${ }^{9} 1031$ Although interventional strategies are equally effective in biomarker-positive men and women ${ }^{32}$ and the potential benefit on survival after 5 years appears to be similar in both genders, invasive therapy remains less frequently used in women than in men with AMI. ${ }^{33}$ In our study, PCI treatment continuously increased in both genders but was age dependent. Therefore, the age-adjusted mortality decrease in patients with AMI could in large be explained by the increase in reperfusion therapy with no differences between men and women. In a meta-analysis of 35 randomised controlled trials and observational studies of patients with STEMI who underwent PCI, the gender differences in short-term and long-term mortality remained significant even after adjustment. ${ }^{23}$ However, a study from the Mayo Clinic, which included 19239 patients who underwent PCI from 1979 to 2004, showed a decrease in mortality during this period with no differences between men and women. ${ }^{34}$

Younger women with AMI had a higher mortality than men of similar age..$^{12-15}$ Our study showed that the decrease in in-hospital mortality of the patients below 60 years was not significant in male patients but markedly reduced in female patients over the last 20 years. A study on young patients with AMI (30 to 54 years) from the National Inpatients Sample database showed that in-hospital mortality decreased between 2001 and 2010 in women although they had more comorbidities than young men. ${ }^{35}$ We have not seen similar differences in the 
Swiss population. Further studies are therefore needed in order to be able to explain these differences.

Throughout the entire observational period in our study, women were overall less likely to received guideline-recommended drugs, which is in line with other publications. ${ }^{36}$ This is in contrast to the findings of the 'Acute Coronary Treatment and Intervention Outcomes Network Registry-Get with the Guidelines' study of 75370 patients with STEMI and NSTEMI enrolled from July 2008 to March 2011 where acute and discharge medical therapies were similar by race in any gender category. ${ }^{37}$ In patients with NSTEMI, gender-based differences in adverse outcomes persisted even with an increased rate of early invasive therapy. ${ }^{38}$ As the strategy of an early invasive approach for women with NSTEMI was considered controversial in the 2000s, we decided against analysing this particular point.

\section{Limitations of the study}

The limitations of the study are those common to all observational studies, particularly long-lasting studies over decades. The diagnosis of AMI has been repeatedly updated during the last 20 years, and the introduction of new biomarkers has resulted in more patients being diagnosed with AMI, particularly patients with NSTEMI, which is a non-calculated bias. Furthermore, the choice of intervention and medication was at the discretion of the treating physicians or internal hospital guidelines, which could also cause an unknown bias. However, data from 'real-life' routine clinical practice, which includes patients who were polymorbid, who were late comers or who were treated palliative only, contribute to a better estimation of the overall burden of AMI.

\section{CONCLUSIONS}

From 1997 to 2016, in-hospital mortality of patients with AMI in Switzerland has halved and the gender gap was further reduced. Accordingly, the mortality reduction was more pronounced in women, especially those in the age category below 60 years. The mortality decrease could in large be explained by the increase in the use of reperfusion therapy, in particular PCI, whereby the gender difference disappeared.

\section{Acknowledgements We would like to thank Jenny Piket for proof reading this manuscript.}

Contributors DR: conception and design, analysis and interpretation of data, drafting of the article. BS: analysis and interpretation of data, critical revision of manuscript and final approval of the manuscript. MR, PU, HR and GP: acquisition of data, critical revision of manuscript for intellectual content and final approval of the manuscript. PE: acquisition of data, conception and design, critical revision of manuscript for intellectual content and final approval of the manuscript.

Funding The AMIS Plus registry is funded by unrestricted grants from the Swiss Heart Foundation and from Abbot AG, Amgen AG, AstraZeneca AG, Bayer (Schweiz) AG, B. Braun Medical AG, Biotronik AG, Boston Scientific AG, Cordis-Cardinal Health, Daiichi-Sankyo/Eli Lilly AG, GE Healthcare, Medtronic AG, A Menarini AG, Mepha Pharma AG, Merck Sharp \& Dohme-Chibret AG, Novartis Pharma Schweiz AG, Pfizer AG, Servier (Suisse) AG, St. Jude Medical and Vascular Medical AG, all in Switzerland. Disclaimer The sponsors did not play any role in the design, data collection, analysis or interpretation of the registry.

Competing interests None declared.

Ethics approval Swiss Supra-Regional Ethics Committee for Clinical Studies, the Swiss Board for Data Security and the Cantonal Ethics Commissions.

Provenance and peer review Not commissioned; internally peer reviewed.

Data sharing statement According to the AMIS Plus regulations, there are access restrictions to the individual data used as these are the property of the hospitals participating in the AMIS Plus registry and may only be made available by each hospital principal investigator and the AMIS Plus Steering Committee. This also applies to any analysis files or parts thereof used in this study.

Open Access This is an Open Access article distributed in accordance with the Creative Commons Attribution Non Commercial (CC BY-NC 4.0) license, which permits others to distribute, remix, adapt, build upon this work non-commercially, and license their derivative works on different terms, provided the original work is properly cited and the use is non-commercial. See: http://creativecommons.org/ licenses/by-nc/4.0/

(c) Article author(s) (or their employer(s) unless otherwise stated in the text of the article) 2017. All rights reserved. No commercial use is permitted unless otherwise expressly granted.

\section{REFERENCES}

1. Chimonas T, Fanouraki I, Liberopoulos EN, et al. Diverging trends in cardiovascular morbidity and mortality in a low risk population. Eur $J$ Epidemiol 2009;24:415-23.

2. Krumholz HM, Wang Y, Chen J, et al. Reduction in acute myocardial infarction mortality in the United States: risk-standardized mortality rates from 1995-2006. JAMA 2009;302:767-73.

3. Movahed MR, John J, Hashemzadeh M, et al. Mortality trends for non-ST-segment elevation myocardial infarction (NSTEMI) in the United States from 1988 to 2004. Clin Cardiol 2011;34:689-92.

4. Schmidt M, Jacobsen JB, Lash TL, et al. 25 year trends in first time hospitalisation for acute myocardial infarction, subsequent short and long term mortality, and the prognostic impact of sex and comorbidity: a Danish nationwide cohort study. BMJ 2012;344:e356.

5. Smolina K, Wright FL, Rayner M, et al. Long-term survival and recurrence after acute myocardial infarction in England, 2004 to 2010. Circ Cardiovasc Qual Outcomes 2012;5:532-40.

6. Insam C, Paccaud F, Marques-Vidal P. The region makes the difference: disparities in management of acute myocardial infarction within Switzerland. Eur J Prev Cardiol 2014;21:541-8.

7. Wilmot KA, O'Flaherty M, Capewell S, et al. Coronary heart disease mortality declines in the United States from 1979 through 2011: evidence for stagnation in young adults, especially women. Circulation 2015;132:997-1002.

8. Albrektsen G, Heuch I, Løchen ML, et al. Lifelong gender gap in risk of incident myocardial infarction: the Tromso study. JAMA Intern Med 2016;176:1673-9.

9. Radovanovic D, Erne P, Urban P, et al. Gender differences in management and outcomes in patients with acute coronary syndromes: results on 20,290 patients from the AMIS Plus Registry. Heart 2007;93:1369-75.

10. Radovanovic D, Nallamothu BK, Seifert B, et al. Temporal trends in treatment of ST-elevation myocardial infarction among men and women in Switzerland between 1997 and 2011. Eur Heart J Acute Cardiovasc Care 2012;1:183-91.

11. Roffi M, Radovanovic D, Erne P, et al. Gender-related mortality trends among diabetic patients with ST-segment elevation myocardial infarction: insights from a nationwide registry 1997-2010. Eur Heart J Acute Cardiovasc Care 2013;2:342-9.

12. Champney KP, Frederick PD, Bueno $\mathrm{H}$, et al. The joint contribution of sex, age and type of myocardial infarction on hospital mortality following acute myocardial infarction. Heart 2009;95:895-9.

13. Khera S, Kolte D, Gupta T, et al. Temporal trends and sex differences in revascularization and outcomes of st-segment elevation myocardial infarction in younger adults in the United States. J Am Coll Cardiol 2015;66:1961-72.

14. Idris N, Aznal SS, Chin SP, et al. Acute coronary syndrome in women of reproductive age. Int J Womens Health 2011;3:375-80.

15. Otten AM, Maas AH, Ottervanger JP, et al. Is the difference in outcome between men and women treated by primary percutaneous coronary intervention age dependent? Gender difference in STEMI stratified on age. Eur Heart J Acute Cardiovasc Care 2013;2:334-41. 
16. Mehta LS, Beckie TM, DeVon HA, et al. Acute myocardial infarction in women: a scientific statement from the american heart association. Circulation 2016;133:916-47.

17. Radovanovic D, Erne P. AMIS Plus: Swiss registry of acute coronary syndrome. Heart 2010;96:917-21.

18. Jeger RV, Radovanovic D, Hunziker PR, et al. Ten-year trends in the incidence and treatment of cardiogenic shock. Ann Intern Med 2008;149:618-26.

19. Schoenenberger AW, Radovanovic D, Stauffer JC, et al. Age-related differences in the use of guideline-recommended medical and interventional therapies for acute coronary syndromes: a cohort study. J Am Geriatr Soc 2008;56:510-6.

20. Erne P, Bertel O, Urban P, et al. Inpatient versus outpatient onsets of acute myocardial infarction. Eur J Intern Med 2015;26:414-9.

21. Thygesen K, Alpert JS, White HD. Joint ESC/ACCF/AHA/WHF Task Force for the Redefinition of Myocardial Infarction. Universal definition of myocardial infarction. Eur Heart J 2007;28:2525-38.

22. Poon S, Goodman SG, Yan RT, et al. Bridging the gender gap: insights from a contemporary analysis of sex-related differences in the treatment and outcomes of patients with acute coronary syndromes. Am Heart J 2012;163:66-73.

23. Pancholy SB, Shantha GP, Patel T, et al. Sex differences in shortterm and long-term all-cause mortality among patients with ST-segment elevation myocardial infarction treated by primary percutaneous intervention: a meta-analysis. JAMA Intern Med 2014:174:1822-30.

24. Halvorsen S, Eritsland J, Abdelnoor M, et al. Gender differences in management and outcome of acute myocardial infarctions treated in 2006-2007. Cardiology 2009;114:83-8.

25. Jneid H, Fonarow GC, Cannon CP, et al. Sex differences in medical care and early death after acute myocardial infarction. Circulation 2008;118:2803-10.

26. Radovanovic D, Auer M, Erne P. Sex-related differences in treatment and outcome in patients with acute coronary syndrome. Cardiol Rev 2008;25:21-6.

27. Valero-Masa MJ, Velásquez-Rodríguez J, Diez-Delhoyo F, et al. Sex differences in acute myocardial infarction: is it only the age? Int $J$ Cardiol 2017;231:36-41.
28. Woo JS, Kim W, Ha SJ, Sj H, et al. Impact of gender differences on long-term outcomes after successful percutaneous coronary intervention in patients with acute myocardial infarction. Int J Cardiol 2010;145:516-8.

29. Milcent C, Dormont B, Durand-Zaleski I, et al. Gender differences in hospital mortality and use of percutaneous coronary intervention in acute myocardial infarction: microsimulation analysis of the 1999 nationwide French hospitals database. Circulation 2007;115:833-9.

30. Vaccarino V, Parsons L, Peterson ED, et al. Sex differences in mortality after acute myocardial infarction: changes from 1994 to 2006. Arch Intern Med 2009;169:1767-74.

31. Radovanovic D, Erne P. Gender difference in the application of reperfusion therapy in patients with acute myocardial infarction. Cardiology 2009;114:164-6.

32. Shaw LJ, Bugiardini R, Merz CN. Women and ischemic heart disease: evolving knowledge. J Am Coll Cardiol 2009;54:1561-75.

33. Donataccio MP, Puymirat E, Parapid B, et al. In-hospital outcomes and long-term mortality according to sex and management strategy in acute myocardial infarction. Insights from the French ST-elevation and non-ST-elevation Myocardial Infarction (FAST-MI) 2005 Registry. Int J Cardiol 2015;201:265-70.

34. Singh M, Rihal CS, Gersh BJ, et al. Mortality differences between men and women after percutaneous coronary interventions. A 25year, single-center experience. J Am Coll Cardiol 2008;51:2313-20.

35. Gupta A, Wang Y, Spertus JA, et al. Trends in acute myocardial infarction in young patients and differences by sex and race, 2001 to 2010. J Am Coll Cardiol 2014;64:337-45.

36. Nauta ST, Deckers JW, van Domburg RT, et al. Sex-related trends in mortality in hospitalized men and women after myocardial infarction between 1985 and 2008: equal benefit for women and men. Circulation 2012:126:2184-9.

37. Edmund Anstey D, Li S, Thomas L, et al. Race and sex differences in management and outcomes of patients after ST-elevation and nonST-elevation myocardial infarct: results from the ncdr. Clin Cardiol 2016;39:585-95.

38. Udell JA, Koh M, Qiu F, et al. Outcomes of women and men with acute coronary syndrome treated with and without percutaneous coronary revascularization. J Am Heart Assoc 2017;6:e004319. 